\author{
GENERAL INFORMATION \\ https://www.cambridge.org/core/journals/ \\ canadian-journal-of-mathematics/information
}

The CMS publishes two research journals: the Canadian Journal of Mathematics (CJM) for longer papers, and the Canadian Mathematical Bulletin (CMB) for shorter papers. To be submitted to CJM, papers should be at least 18 pages long. Shorter papers should be submitted to the CMB. All submitted papers are considered subject to the understanding that they have not been published and that they are not being considered for publication elsewhere. To be accepted, papers must treat new mathematical research, be well written, and be of interest to a significant segment of the mathematical community. Papers may be written in English or French. Manuscripts must be prepared in accordance with the instructions for authors provided at

https://www.cambridge.org/core/journals/canadian-journalof-mathematics/information/information-for-author

\section{SUBSCRIPTION INFORMATION}

Subscription rates can be found at

https://www.cambridge.org/core/journals/canadian-journalof-mathematics/subscribe

Members of the CMS are entitled to a reduced subscription rate. For more information, please contact the CMS at memberships@cms.math.ca.

\section{CANADA POSTMASTER}

Canadian Journal of Mathematics

Publications Mail Registration: 40005057

Send address changes to:

Journals Fulfillment Department, Cambridge University Press, One Liberty Plaza, New York, NY 10006 USA.

\section{CANADIAN JOURNAL OF MATHEMATICS}

(USPS \# 010-741) is published six times per year, bimonthly (February, April, June, August, October, December). Published by Cambridge University Press, One Liberty Plaza, New York, NY 10006 USA on behalf of the Canadian Mathematical Society. US Mailing Agent: Sheridan Press, 450 Fame Ave., Hanover, PA 17331 USA.

Periodicals postage paid at New York, NY and at additional mailing offices.

US POSTMASTER: Send address changes to Journals Fulfillment Department, Cambridge University Press, One Liberty Plaza, New York, NY 10006 USA.

\author{
RENSEIGNEMENTS GÉNÉRAUX \\ https://www.cambridge.org/core/journals/ \\ canadian-journal-of-mathematics/information
}

La SMC publie deux revues de recherche: le Journal canadien de mathématiques (JCM) qui présente des articles longs et le Bulletin canadien de mathématiques (BCM) des articles plus courts. Les articles destinés au JCM doivent compter au moins 18 pages. Les articles plus courts doivent être acheminés au BCM. Il est entendue que les articles présentés pour publication sont inédits et qu'ils ne sont pas à l'étude pour publication dans d'autres revues. Un article accepté porte sur des recherches récentes, est bien rédigé et s'avère susceptible d'intéresser bon nombre de mathématiciens. Nous acceptons les articles rédigés en langues anglaise ou française. Les articles doivent répondre aux conventions énoncés pour les auteurs au https://www.cambridge.org/core/journals/canadian-journalof-mathematics/information/information-for-author

\section{ABONNEMENTS}

Les tarifs d'abonnements sont disponibles au https://www.cambridge.org/core/journals/canadian-journalof-mathematics/subscribe

Les membre de la SMC ont droits à un taux d'abonnements réduits. Pour plus amples renseignements, veuillez contacter la SMC à adhesions@smc.math.ca.

\section{POSTES CANADIENNES}

Journal canadien de mathématiques

No. d'enregistrement du courrier publications: 40005057

Envoyer les changements d'adresses aux:

Journals Fulfillment Department, Cambridge University Press, One Liberty Plaza, New York, NY 10006 USA.

JOURNAL CANADIEN DE MATHEMATIQUES (USPS \# 010-741) est publié six fois par an, bimensuel (février, avril, juin, août, octobre, décembre). Publié par Cambridge University Press, One Liberty Plaza, New York, NY 10006 USA au nom de la Société mathématique du Canada. Agent de poste US: Sheridan Press, 450 Fame Ave., Hanover, PA 17331 USA.

Postes payé à New York, et autres bureaux de diffusion supplémentaires.

POSTES AMÉRICAINES: Envoyer les changements d'adresses aux Journals Fulfillment Department, Cambridge University Press, One Liberty Plaza, New York, NY 10006 USA. 


\section{In this issue / Dans ce numéro}

Isospectrality for Orbifold Lens Spaces

326

373

392

427

455

480

505

537 Counterpart

Marginals with Finite Repulsive Cost and Quartic Hecke $L$-functions Problem for the $f$-divergence

Varieties and Schreieder Varieties Bound Theorems
Newforms of Half-integral Weight: The Minus Space

Automatic Sequences and Generalised Polynomials

One-Level Density of Low-lying Zeros of Quadratic

Orlicz Addition for Measures and an Optimization

Primes Dividing Invariants of CM Picard Curves

On the Chow Ring of Cynk-Hulek Calabi-Yau

Almost Simplicial Polytopes: The Lower and Upper

Corrigendum to: A Galois Correspondence for Reduced Crossed Products of Simple $\mathrm{C}^{*}$-algebras by Discrete Groups
Naveed S. Bari,

Eugenie Hunsicker

Ehud Moshe Baruch, Soma Purkait

Ugo Bindini

Jakub Byszewski, Jakub Konieczny

Peng Gao,

Liangyi Zhao

Shaoxiong Hou,

Deping Ye

Pınar Kilıçer, Elisa Lorenzo García, Marco Streng

Robert Laterveer,

Charles Vial

Eran Nevo,

Guillermo Pineda-Villavicencio, Julien Ugon, David Yost

Jan Cameron, Roger R. Smith 